\title{
Immobilization of a carbon nanomaterial-based localized drug-release system using a bispecific material-binding peptide
}

This article was published in the following Dove Press journal:

International Journal of Nanomedicine

\section{Katsutoshi Kokubun ${ }^{1,2}$ \\ Sachiko Matsumura' \\ Masako Yudasaka ${ }^{3,4}$ \\ Sumio lijima ${ }^{3,4}$ \\ Kiyotaka Shiba'}

'Division of Protein Engineering, Cancer Institute, Japanese Foundation for Cancer Research, Tokyo, Japan; ${ }^{2}$ Department of Clinical Pathophysiology, Tokyo Dental College, Tokyo, Japan; ${ }^{3}$ Nanomaterials Research Institute, National Institute of Advanced Industrial Science and Technology, Tsukuba, Japan; ${ }^{4}$ Graduate School of Science and Technology, Meijo University, Nagoya, Japan
Correspondence: Katsutoshi Kokubun Department of Clinical Pathophysiology, Tokyo Dental College, 2-9-18

Kandamisaki-cho, Chiyoda-ku, Tokyo, I0I-006I, Japan

$\mathrm{Tel}+8 I 363809252$

$\mathrm{Fax}+81363809606$

Email kkokubun@tdc.ac.jp
Introduction: Inorganic materials are widely used in medical devices, such as artificial hearts, vessels, and joints, in stents, and as nanocarriers for drug-delivery systems. Carbon nanomaterials are of particular interest due to their biological inertness and their capability to accommodate molecules. Several attempts have been proposed, in which carbon nanomaterials are used as nanocarriers for the systemic delivery of drugs.

Materials and methods: We developed a drug-delivery system in which oxidized singlewalled carbon nanohorns (oxSWNHs) were immobilized on a titanium (Ti) surface using material-binding peptides to enable localized drug delivery. For this purpose, we utilized a bispecific peptidic aptamer comprising a core sequence of a Ti-binding peptide and a SWNHbinding peptide to immobilize oxSWNHs on Ti.

Results: Scanning electron microscopy was used to confirm the presence of oxSWNHs adsorbed onto the Ti surface, and a quartz crystal microbalance was used to evaluate the binding process during oxSWNH adsorption. The oxSWNHs-ornamented Ti substrate was nontoxic to cells and released biologically active dexamethasone over a sustained period.

Conclusion: This oxSWNHs-immobilized system can be used to modify the surface of Ti in implants and be loaded with drugs that stimulate osteogenesis and bone regeneration.

Keywords: drug carrier, drug delivery, carbon nanomaterial, carbon nanohorn, peptide aptamer

\section{Introduction}

Controlled surface modification of substrates is critical for the development of biomaterials that can interact with cells. ${ }^{1-4}$ Surface modifications can be classified as physical, chemical, or biological. ${ }^{5}$ In the chemical approach, various types of drug compounds are used as components of modifiers, which induce highly specific reactions in the biological system. To develop a functionalized surface that can release drugs into the microenvironment adjacent to the materials, we must first establish a system to load the drug and slowly release it from the carrier.

Titanium (Ti) is widely used as a biomaterial for dental implants, artificial joints, and stents. ${ }^{6}$ For developing next-generation titanium implants, several modification methods have been proposed to reduce the time required for osseointegration. These methods include the use of sintered metal beads, surface blasting, acid or alkaline treatment, anodization, and ion implantation. ${ }^{7-10}$ Surface chemistry is a pivotal factor in the regulation of interactions between the biomaterial and cells/tissue. ${ }^{11-14}$ In addition to these physical and chemical methods, modification using natural proteins, such as collagen, cell-adhesive molecules, or growth factors, has also been attempted. ${ }^{5}$ For example, Kashiwagi et $\mathrm{al}^{3}$ ornamented Ti surfaces with modified bone-morphogenetic protein 
(BMP) that had an affinity for Ti. Different methodologies have been designed to control drug release from Ti. ${ }^{15,16}$ One strategy relies on surface modification. Usually, the rough Ti surface contributes to enhanced drug loading as compared with plane Ti. For example, a Ti plate containing nanopores shows enhanced drug-loading capability. ${ }^{17}$ Another approach involves attachment of therapeutic molecules directly to the Ti surface. Therapeutic molecules are chemically attached to a pure Ti surface ${ }^{18}$ or to self-assembled monolayers, which act as a glue. ${ }^{19}$ Additionally, drug coatings comprising drugs and hydroxyapatite ${ }^{20}$ or polymer ${ }^{21}$ show controlled drug-release profiles in vitro and in vivo.

Carbon nanomaterials have potential applications as carriers in drug-delivery systems ${ }^{22-29}$ because of their favorable characteristics, including stability, inertness, and a large surface area for drug loading. ${ }^{30-33}$ A single-walled carbon nanohorn $(\mathrm{SWNH})$ is a spherical aggregate with a diameter of $\sim 50 \mathrm{~nm}$ to $\sim 100 \mathrm{~nm}$ and comprising $\sim 2,000$ single-walled graphene tubules with a nonuniform diameter of $2 \mathrm{~nm}$ to $5 \mathrm{~nm}$ and a length of $40 \mathrm{~nm}$ to $50 \mathrm{~nm} .{ }^{34}$ SWNHs have an extensive surface area and intertubule interstices that can accommodate large amounts of adsorbed molecules. ${ }^{35}$ Moreover, the surface area can be enlarged by oxidization, which causes the formation of nanowindows in the SWNH walls, ${ }^{36}$ through which a variety of small molecules can infiltrate into the inner space. ${ }^{37}$ Oxidized SWNHs (oxSWNHs) can open two to four holes that allow the passage of various materials, including drugs, which can then be stored inside the aggregate. ${ }^{37,38}$ The ability of oxSWNHs to load and release drugs, such as dexamethasone (DEX), ${ }^{28}$ doxorubicin, ${ }^{39}$ and cisplatin, ${ }^{40,41}$ has also been shown, and no acute toxicity has been reported for SWNHs in animals, ${ }^{33,42,43}$ highlighting their utility for biological applications.

In this study, we used a Ti-binding peptide (TBP-1 $)^{44}$ and SWNH-binding peptide (NHBP-1 ${ }^{45}$ to immobilize a drugdelivery system on a Ti surface. TBP-1 is a material-binding peptide isolated as an aptamer against Ti. Although originally isolated as a 12-mer peptide (RKLPDAPGMHTW), further mutational analyses revealed that a hexapeptide sequence (RKLPDA; named minTBP-1) is sufficient to mediate binding. The arginine, proline, and aspartate in this sequence (R1, P4, and D5, respectively) are critical for Ti recognition, suggesting that electrostatic interactions constitute the predominant binding force. ${ }^{46}$ NHBP-1 is a 12 -amino acid aptamer (DYFSSPYYEQLF) isolated as a specific SWNH binder using a peptide phage system. Non-covalent modification with a conjugate of polyethylene glycol and NHBP-1 enhances the dispersibility of hydrophobic oxSWNHs under aqueous conditions. ${ }^{41}$
Recent studies of peptide aptamers have highlighted the importance of valency on the part of the displayed peptide for target affinity with the conjugate. Multivalent display of peptide aptamers increases the affinity of the conjugate for the target, ${ }^{47,48}$ and in one instance, multivalency increased conjugate adsorption. ${ }^{2}$ We speculated that TBP-NHBP generated by fusing minTBP-1 and NHBP-1 would be adsorbed onto oxSWNHs, enabling a multivalent display of minTBP-1 on oxSWNHs and allowing the latter to be adsorbed onto the $\mathrm{Ti}$ surface. To test this possibility, we synthesized TBP-NHBP and developed a method for immobilizing oxSWNHs on the Ti surface using this peptide. We also tested the ability of DEX-loaded oxSWNHs immobilized on a Ti surface to release the drug in a sustained fashion and examined how this affects the activation of preosteoblasts grown on the functionalized Ti surface.

\section{Materials and methods Adsorption of oxSWNHs onto Ti plates}

oxSWNHs were prepared by oxidation of as-grown SWNHs in dry air, with the temperature increased from room temperature to $550^{\circ} \mathrm{C}$ at a rate of $1^{\circ} \mathrm{C} / \mathrm{min} .{ }^{49}$ oxSWNHs were dispersed in dimethylformamide (DMF) at $10 \mathrm{mg} / \mathrm{mL}$, and after sonication at $130 \mathrm{~W}$ (Bioruptor ${ }^{\circledR} \mathrm{UCD}^{2} 200$; Cosmo Bio Co. Ltd, Tokyo, Japan), $5 \mu \mathrm{L}$ of the oxSWNH suspension was diluted by adding $175 \mu \mathrm{L}$ of $10 \%$ DMF in water (purified with a Biocel A10 filter; Merck Millipore, Billerica, MA, USA), followed by brief sonication. A $20-\mu \mathrm{L}$ volume of TBP-NHBP (RKLPDAGGDYFSSPYYEQLF; $1 \mathrm{mM}$ in DMF) was added to the solution, which was vortexed for $10 \mathrm{~min}$ at room temperature. TBP-NHBP comprised the peptide aptamers minTBP-1 (RKLPDA) and NHBP-1 (DYFSSPYYEQLF) connected via a GG linker. The custom peptide was constructed via solid-phase peptide synthesis by Anygen Co. Ltd (Gwangju, Korea). The final concentrations of oxSWNHs and TBP-NHBP in the mixture were $0.25 \mathrm{mg} / \mathrm{mL}$ and $100 \mu \mathrm{M}$, respectively. Ti plates (Japan Industrial Specification H4600; 99.9 mass \% Ti, 10×10×1 mm; Shinkinzoku Co. Ltd., Osaka, Japan) were incubated for $1 \mathrm{~h}$ at room temperature in $500 \mu \mathrm{L}$ of the oxSWNHs/TBP-NHBP mixture in 24-well culture plates (BD Biosciences, Sparks, MD, USA), washed three times with water filtered through a $0.22-\mu \mathrm{m}$ membrane (Millipore), and then dried under vacuum for $8 \mathrm{~h}$. The ratio of TBP-NHBP to oxSWNHs was evaluated in pilot experiments under various conditions. oxSWNHs adsorbed onto Ti plates were quantified using an electronic balance (UMX2; Mettler-Toledo, Billerica, MA, USA) and observed by scanning electron microscopy (SEM; VE-8800, Keyence, Osaka, Japan). 


\section{Quartz crystal microbalance (QCM) measurements}

The binding of oxSWNHs to the Ti surface was evaluated with a QCM (QCM-D300; Q-Sense AB, Götenborg, Sweden) and a Ti sputter-coated QCM sensor that was first cleaned for 10 min using an ultraviolet/ozone cleaner surface-treatment system (ProCleaner ${ }^{\mathrm{TM}}$, BioForce Nanosciences, Inc., Salt Lake City, UT, USA). Measurements were performed at $25^{\circ} \mathrm{C}$, and data were collected at 14.8 MHz. The sensor was first equilibrated with the mixture containing oxSWNHs and TBP-NHBP1 peptide $(100 \mu \mathrm{M})$ in 10\% DMF, followed by washing with $0.5 \mathrm{~mL}$ of $10 \% \mathrm{DMF}$. The second and subsequent layers were formed in the same manner. Data were analyzed using Q-tools software (https://qtools.com).

\section{Cell viability}

Cell viability was qualitatively analyzed by fluorescence microscopy. MC3T3-E1 cells (ATCC) were seeded on Ti plates $(10 \times 10 \times 1 \mathrm{~mm})$ in 24 -well plates (BD Biosciences) at a density of $1 \times 10^{5}$ cells/well and cultured for $48 \mathrm{~h}$. MC3T3E-1 cells were maintained in $\alpha$-minimal essential medium ( $\alpha$-MEM; Gibco $^{\circledR}$, Thermo Fisher Scientific, Grand Island, NY, USA) supplemented with $10 \%$ fetal bovine serum (FBS; JRH Bioscience, Lenexa, KS, USA), $100 \mu \mathrm{g} / \mathrm{mL}$ penicillin (Banyu Pharmaceutical Co., Tokyo, Japan), and $100 \mathrm{U} / \mathrm{mL}$ streptomycin (Meiji Seika Kaisha, Tokyo, Japan) in continuous culture at $37^{\circ} \mathrm{C}$ under a humidified atmosphere with $5 \% \mathrm{CO}_{2}$ and $95 \%$ air. After three washes with phosphate-buffered saline (PBS; Gibco-BRL, Carlsbad, CA, USA), adherent cells were stained with $2 \mu \mathrm{M}$ calcein-AM and $4 \mu \mathrm{M}$ propidium iodide (Dojindo Laboratories, Kumamoto, Japan) in PBS and washed twice with PBS. The stained cells were photographed under a DMLP reflection microscope (Leica Microsystems, Bensheim, Germany) equipped with a DC480 or DFC350 FX digital camera.

\section{Adsorption of DEX-oxSWNHs onto Ti plates}

oxSWNHs $(500 \mu \mathrm{g} / \mathrm{mL})$ were dispersed by sonication in $10 \%$ DMF, and cell-culture-grade DEX $(1,000 \mu \mathrm{g} / \mathrm{mL}$; Sigma-Aldrich, St Louis, MO, USA) in 10\% DMF was added at a volumetric ratio of $1: 1$. The mixture was vortexed for $10 \mathrm{~min}$ and incubated overnight at room temperature. DEX-oxSWNHs were mixed with TBP-NHBP and vortexed for $10 \mathrm{~min}$. Ti plates $(10 \times 10 \times 1 \mathrm{~mm})$ in 24 -well culture plates were incubated for $1 \mathrm{~h}$ at room temperature in $500 \mu \mathrm{L}$ of the above mixture, washed three times with water filtered through a $0.22-\mu \mathrm{m}$ membrane (Millex-GS; Millipore), and dried under vacuum for $8 \mathrm{~h}$. The amount of DEX was quantified with $\left[{ }^{3} \mathrm{H}\right]-\mathrm{DEX}(1.48 \mathrm{TBq} / \mathrm{mmol}$; Amersham Bioscience,
Piscataway, NJ, USA) using an LS6500 scintillation counter (Beckman Coulter, Fullerton, CA, USA).

\section{Analysis of in vitro release of DEX from DEX-oxSWNHs on a Ti surface}

DEX-oxSWNHs were prepared as described using $\left[{ }^{3} \mathrm{H}\right]-$ DEX and incubated at $37^{\circ} \mathrm{C}$ in PBS, Roswell Park Memorial Institute (RPMI) 1640 medium (Gibco), or $\alpha$-MEM supplemented with $5 \% \mathrm{FBS}, 100 \mu \mathrm{g} / \mathrm{mL}$ penicillin, and $100 \mathrm{U} / \mathrm{mL}$ streptomycin. Samples were collected at predetermined time points, and the amount of $\left[{ }^{3} \mathrm{H}\right]$-DEX released into the supernatant was quantified using a scintillation counter. For cumulative-release experiments, the supernatant was replaced with fresh medium at each time point.

\section{DEX-responsive promoter assay}

An expression vector containing the luciferase-reporter gene under the control of a DEX-responsive element (pBV2MMTV-LUC) was gifted by Drs H Noguchi and I Abe of the University of Shizuoka (Shizuoka, Japan). ${ }^{50}$ ST2 cells (RIKEN BioResource Center) were seeded at a density of $2.5 \times 10^{4}$ cells/well on Ti plates $(10 \times 10 \times 1 \mathrm{~mm})$ in 24 -well plates. ST2 cells were maintained in RPMI 1640 supplemented with $10 \% \mathrm{FBS}, 100 \mu \mathrm{g} / \mathrm{mL}$ penicillin, and $100 \mathrm{U} / \mathrm{mL}$ streptomycin in continuous culture at $37^{\circ} \mathrm{C}$ under a humidified atmosphere with $5 \% \mathrm{CO}_{2}$ and $95 \%$ air. After incubating for 1 day, cells were co-transfected with pBV2-MMTV-LUC and thymidine kinase promoter-driven Renilla luciferase DNA (Promega Corporation, Fitchburg, WI, USA) using FuGENE6 (Promega). After an additional $24 \mathrm{~h}$, luciferase activity was measured using the dual-luciferase reporter assay system (Promega) and an AutoLumat LB953 luminometer (Berthold Technologies, Oak Ridge, TN, USA) according to the manufacturer's instructions.

\section{Alkaline phosphatase (ALP) assay}

MC3T3-E1 cells were seeded at a density of $1.0 \times 10^{4}$ cells/ well on Ti plates $(10 \times 10 \times 1 \mathrm{~mm})$ in 24 -well plates and grown until they reached $70 \%-80 \%$ confluence. The culture medium was changed to a differentiation-inducing medium consisting of $\alpha$-MEM supplemented with 5\% FBS, $100 \mathrm{U} / \mathrm{mL}$ penicillin, $100 \mu \mathrm{g} / \mathrm{mL}$ streptomycin, $50 \mu \mathrm{M}$ ascorbic acid, $10 \mathrm{mM}$ $\beta$-glycerophosphate, and $20 \mathrm{ng} / \mathrm{mL}$ recombinant human bone morphogenetic protein-4 (Genzyme/TECHNE, Cambridge, MA, USA). The cells were incubated for 5, 7, 10, or 14 days, during which time the medium was refreshed every 3 days. Medium changes were performed carefully to ensure that oxSWNHs or DEX-oxSWNHs adhering to the Ti plates were not disturbed and removed along with the medium. 
To evaluate ALP activity, the medium was removed, and cells were washed three times with Tris-buffered saline (TBS) comprising $20 \mathrm{mM}$ Tris (pH 7.4) and $150 \mathrm{mM} \mathrm{NaCl}$. The cells were harvested by scraping them into $200 \mu \mathrm{L}$ TBS containing $1 \%$ Triton X-100 on ice and sonicated on ice for $5 \mathrm{~min}$. The resulting cell lysates were centrifuged at $16,100 \mathrm{~g}$ for $20 \mathrm{~min}$ at $4^{\circ} \mathrm{C}$, and the supernatant $(50 \mu \mathrm{L})$ was mixed with $p$-nitrophosphate. The amount of $p$-nitrophenol released after 45 min was measured by spectrophotometry (405 nm) using a Model 550 microplate reader (Bio-Rad Laboratories Inc., Hercules, CA, USA). The protein content of the supernatant was assayed by the Lowry method using a bicinchoninic acid protein assay kit (Pierce, Rockford, IL, USA). ALP activity was expressed as the amount of released $p$-nitrophenol normalized to total protein concentration.

\section{Statistical analysis}

Differences between groups were evaluated by one-way analysis of variance. Any $P$-values $<0.05$ and $<0.01$ were considered significant.

\section{Results}

\section{Immobilization of oxSWNHs/TBP-NHBP on a $\mathrm{Ti}$ surface}

We generated TBP-NHBP(RKLPDAGGDYFSSPYYEQLF), a fusion of minTBP-1 and NHBP-1 connected via a GG linker, to mediate the binding of oxSWNHs to the Ti surface (Figure 1). TBP-NHBP was mixed with oxSWNHs in 10\% DMF to obtain the dispersed oxSWNHs/TBP-NHBP complex, which was adsorbed onto the Ti surface by incubating Ti plates in dispersed solutions of the complex. Three samples [oxSWNHs without peptide (oxSWNHs), a mixture of minTBP-1 and oxSWNHs (oxSWNHs/TBP), and a mixture of NHBP and oxSWNHs (oxSWNHs/NHBP)] were adsorbed onto the Ti plates and served as the controls. Figure $2 \mathrm{~A}$ shows the SEM image of the oxSWNHs/TBP-NHBP-adsorbed

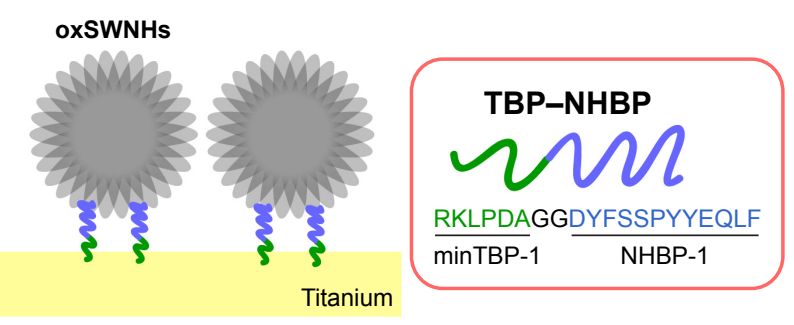

Figure I Schematic representation of oxSWNHs containing the TBP-NHBP peptide complex (oxSWNHs/TBP-NHBP) on the Ti surface. oxSWNHs were modified by artificial peptide aptamers against SWNH (NHBP-I) and Ti (minTBP-I).

Abbreviations: oxSWNHs, oxidized single-walled carbon nanohorns; TBP, Ti-binding peptide; NHBP-I, SWNH-binding peptide. particles on the Ti surface. When oxSWNHs were adsorbed onto Ti plates with TBP-NHBP, the weight of the plate increased relative to the controls (Figure 2F). By contrast, the adsorption of oxSWNHs without TBP-NHBP onto the Ti surface was negligible (Figure $2 \mathrm{~B}$ and $\mathrm{F}$ ). When oxSWNHs alone were incubated with the Ti plates, aggregates formed that were nonuniformly adsorbed on the plate surface (Figure 2B). Additionally, we used a TBP-NHBP variant containing an arginine $(\mathrm{R})$ to alanine $(\mathrm{A})$ mutation in the minTBP-1 sequence AKLPDA [TBP(R-A)-NHBP] to determine whether the minTBP-1 sequence in TBP-NHBP was effective for Ti binding. oxSWNHs did not uniformly adsorb to Ti in the presence of TBP(R-A)-NHBP (Figure 2C and $\mathrm{F}$ ), consistent with a previous report showing that loss of the charged $\mathrm{R}$ residue reduced the binding of minTBP-1 to $\mathrm{Ti}^{44,47}$ Additionally, the dispersibility of oxSWNHs might have been reduced by the R-to-A substitution. These results indicated that the minTBP-1 sequence promoted binding of oxSWNHs to Ti. Additionally, oxSWNHs did not uniformly adsorb to the Ti surface when mixed with TBP or NHBP alone (Figure 2D-F), indicating that TBP-NHBP conferred the binding characteristics of minTBP-1 on oxSWNHs.

To investigate the ability of TBP-NHBP to promote adsorption of oxSWNHs onto the Ti surface, we used a QCM to monitor changes in the resonance frequency $(f)$ and dissipation $(D)$ of the Ti plate. Initial infusion of an oxSWNHs/TBP-NHBP solution into the QCM measurement cell reduced $f$ by $\sim 65 \mathrm{~Hz}$, with this response plateauing within $1 \mathrm{~min}$ and ameliorated by subsequent infusion of a wash buffer, indicating that oxSWNHs/TBP-NHBP were bound to the Ti surface (Figure $3 \mathrm{~A}$ ). When the cell chamber was refilled with an oxSWNHs/TBP-NHBP solution, $f$ declined, whereas $D$ increased. By contrast, injection of oxSWNHs alone reduced $f$ by only $9 \mathrm{~Hz}$, indicating a weak association with the Ti sensor (Figure 3B).

We then investigated whether oxSWNHs must be premixed with TBP-NHBP for efficient adsorption to Ti by testing whether pre-adsorption of TBP-NHBP alone to Ti facilitated oxSWNH immobilization through interaction of the latter with the NHBP part of the peptide on the solid phase. To this end, we injected TBP-NHBP alone onto Ti plates, followed by injection of oxSWNHs. This did not result in adsorption of oxSWNHs to Ti (Figure $3 \mathrm{C}$ ), and moreover, there was no adsorption by injection of oxSWNHs after oxSWNHs/TBP-NHBP were injected and allowed to adsorb to the Ti sensor (Figure 3D). These results demonstrated the necessity of pre-mixing TBP-NHBP and oxSWNHs for adsorption of oxSWNHs onto the Ti surface. 

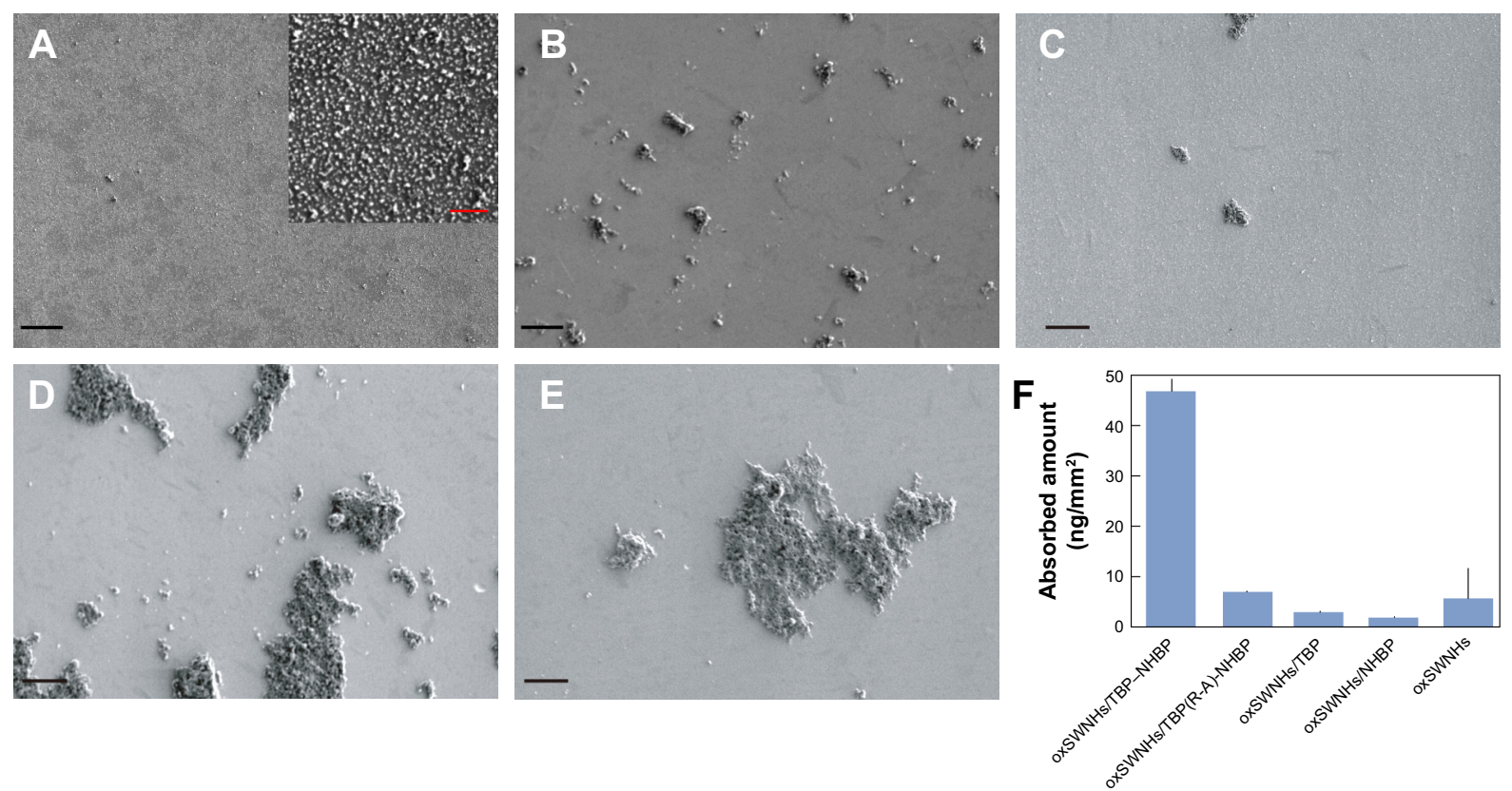

Figure 2 Scanning electron micrographs of Ti plates functionalized with oxSWNHs/TBP-NHBP. (A-E) Ti plates were functionalized with oxSWNHs/TBP-NHBP (A), oxSWNHs (B), oxSWNHs/TBP(R-A)-NHBP (C), oxSWNHs/TBP (D), or oxSWNHs/NHBP (E). The inset shows magnified sections. Black scale: $20 \mu \mathrm{m}$; red scale: $2 \mu \mathrm{m}$. (F) Adsorption amounts measured using an electronic balance.

Abbreviations: oxSWNHs, oxidized single-walled carbon nanohorns; TBP, Ti-binding peptide; NHBP-I, SWNH-binding peptide.

When TBP-NHBP alone was used, adsorption onto Ti plates was observed (Figure 3E); however, use of oxSWNHs/ TBP-NHBP resulted in an increase in $D$ (Figure $3 \mathrm{~F}$ ). Even if cleaning was performed prior to each flow of oxSWNHs/ TBP-NHBP, an increase in $D$ was observed, although when TBP-NHBP only was used, a minimal increase in $D$ was observed. It is possible that this resulted due to the unstable adsorption of some of the oxSWNHs to $\mathrm{Ti}$ in the chamber. Therefore, the minTBP-1 portion of TBP-NHBP also contributed to the immobilization of oxSWNHs on the Ti surface.

We then investigated whether oxSWNHs/TBP-NHBP on $\mathrm{Ti}$ was toxic to cells by culturing MC3T3-E1 cells on oxSWNHs/TBP-NHBP on the Ti plates. The live/dead assay using calcein/propidium-iodide staining revealed good cell viability after 2 days of culture (Figure 4A and B). These results demonstrated that uncoated $\mathrm{Ti}$ and oxSWNHs/ TBP-NHBP on Ti exhibited comparable effects on cell viability. SWNHs do not contain contaminating metals, as they are prepared by laser ablation from pure graphite without a metallic catalyst, which reduces the risk of toxicity. ${ }^{33}$

\section{In vitro release of DEX from DEX- oxSWNHs/TBP-NHBP on Ti}

Controlled release from a drug-carrier complex is an important property of drug-delivery systems. In vitro binding and release of the anti-inflammatory glucocorticoid DEX by
oxSWNHs was previously reported. ${ }^{28}$ Here, we evaluated the in vitro drug-release potential of oxSWNHs on Ti according to the cumulative doses of $\left[{ }^{3} \mathrm{H}\right]$-DEX released from DEXoxSWNHs on Ti plates in PBS, $\alpha$-MEM, and RPMI medium at $37^{\circ} \mathrm{C}$ (Figure 5). The initial release of DEX from Ti was slower in $\alpha$-MEM or RPMI medium than in PBS. Additionally, DEX from Ti in $\alpha$-MEM or RPMI medium was released in a sustained fashion for up to 2 weeks.

\section{Biological activity of DEX released from DEX-oxSWNHs/TBP-NHBP on Ti}

DEX is a synthetic agonist of glucocorticoid receptor (GR), which is a nuclear receptor that binds to glucocorticoid response elements (GREs) to activate target-gene transcription. ${ }^{51}$ We examined the responsiveness of GREs to DEX released from DEX-oxSWNHs/TBP-NHBP on Ti using the reporter plasmid pBV2-MMTV-LUC containing a luciferase gene under the control of GREs. ${ }^{50}$ To this end, we transfected ST2 cells with pBV2-MMTV-LUC, followed by incubation with DEX-oxSWNHs/TBP-NHBP, DEX, or oxSWNHs/TBP-NHBP on Ti. DEX and DEX-oxSWNHs/ TBP-NHBP activated luciferase expression, with the latter having a greater effect, whereas the cells alone and oxSWNHs/ TBP-NHBP had no effect (Figure 6). Additionally, DEXoxSWNHs/TBP-NHBP showed an even greater activation than DEX alone on Ti. 

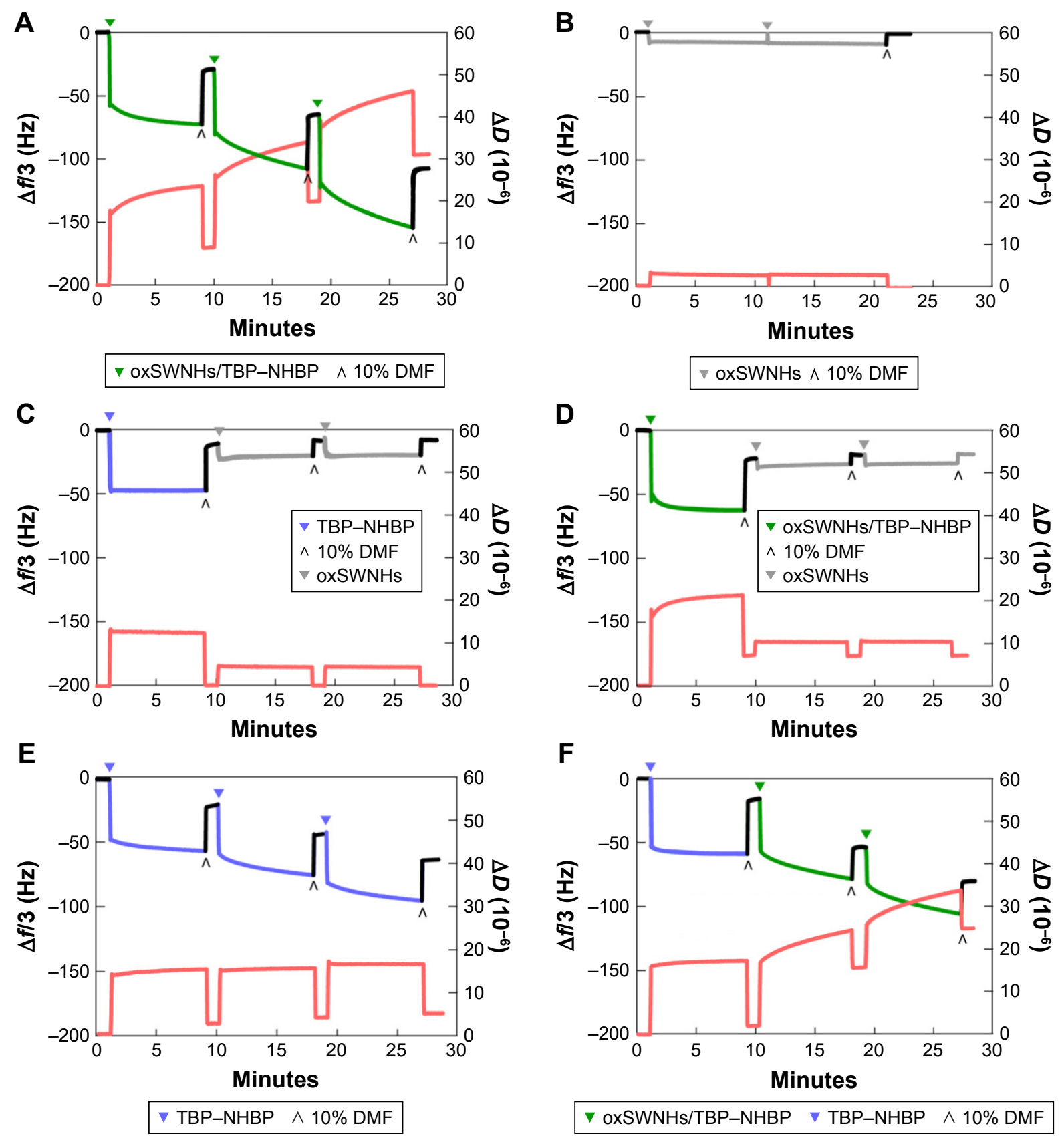

$\nabla$ oxSWNHs/TBP-NHBP $\quad \nabla$ TBP-NHBP $\wedge 10 \%$ DMF

Figure 3 Time-dependent changes in $f$ and $D$ determined using a QCM. (A-F) oxSWNHs/TBP-NHBP (A), oxSWNHs (B), TBP-NHBP (C), oxSWNHs/TBP-NHBP (D), TBP-NHBP (E), and TBP-NHBP and oxSWNHs/TBP-NHBP (F) deposited on a Ti-coated sensor. Values on the longitudinal axis represent negative $\Delta f$ and positive $\Delta D$ values. Changes associated with oxSWNHs/TBP-NHBP, oxSWNHs, and TBP-NHBP adsorption are indicated by green, gray, and purple lines, respectively. Green, gray, and purple arrowheads indicate the time points at which oxSWNHs/TBP-NHBP, oxSWNHs, and TBP-NHBP, respectively, were infused into the measurement chamber. Vs represent the time points at which the wash solution (I0\% DMF) was infused.

Abbreviations: oxSWNHs, oxidized single-walled carbon nanohorns; TBP, Ti-binding peptide; NHBP-I, SWNH-binding peptide; DMF, dimethylformamide.

DEX promotes the differentiation of preosteoblasts into mature osteoblasts, a process characterized by ALP expression..$^{52}$ Additionally, preosteoblastic MC3T3-E1 cells treated with DEX show upregulated expression of ALP, as well as BMP-4, which is a potent inducer of osteoblast differentiation and bone formation. ${ }^{53,54} \mathrm{Here}$, we found that treatment of MC3T3-E1 cells with DEX-oxSWNHs/TBP-NHBP adsorbed on Ti in the presence of BMP-4 increased ALP expression from day 5 to day 14, whereas oxSWNHs/ TBP-NHBP had no effect (Figure 7). Moreover, on day 14, the activity was higher for DEX-oxSWNHs/TBP-NHBP adsorbed on Ti as compared with that observed in control samples $(P<0.05)$. Our results indicated that DEX released from DEX-oxSWNHs on Ti plates was biologically active. 

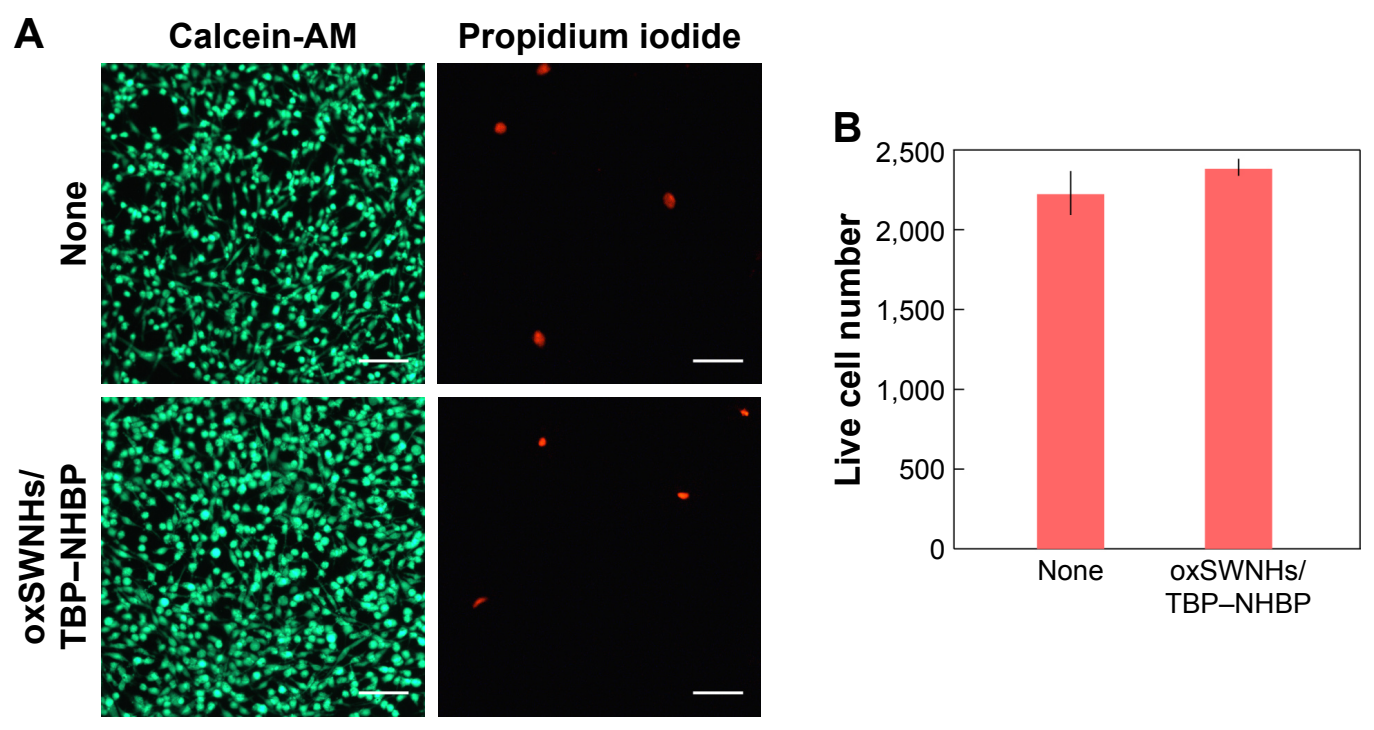

Figure 4 Viability of MC3T3-El cells cultured for 2 days on oxSWNHs/TBP-NHBP on Ti. Viable cell counts were determined relative to cells grown on uncoated Ti (none). (A) Live (green, left panel) and dead (red, right panel) cells were stained with calcein-AM and propidium iodide, respectively. White scale: $100 \mu \mathrm{m}$. (B) Counts of live cells on uncoated Ti and oxSWNHs/TBP-NHBP-conjugated Ti. Error bars indicate standard deviation $(n=4)$.

Abbreviations: oxSWNHs, oxidized single-walled carbon nanohorns; TBP, Ti-binding peptide; NHBP-I, SWNH-binding peptide.

\section{Discussion}

$\mathrm{Ti}$ and its alloys are frequently used for bone and dental implants, and their junction with adjacent bone tissue is paramount. Ti-based materials are also used as scaffolds for biomolecules that control cellular activities, such as adhesion, differentiation, and proliferation..$^{5}$ Ti implants should ideally have osteoinduction capacity, representing the ability to stimulate the formation of new bone from progenitor cells.

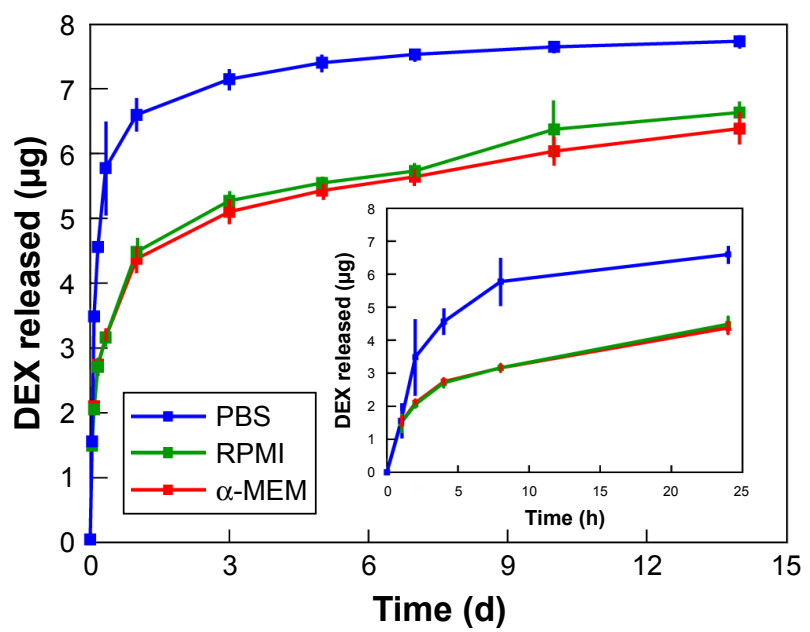

Figure 5 In vitro release of DEX from DEX-oxSWNHs/TBP-NHBP on Ti. Time course of cumulative release of $\left[{ }^{3} \mathrm{H}\right]$-DEX from DEX-oxSWNHs/TBP-NHBP on Ti plates in PBS (blue line), RPMI medium $/ 5 \%$ FBS (green line), and $\alpha-M E M / 5 \% \mathrm{FBS}$ (red line) at $37^{\circ} \mathrm{C}$ over 14 days. The inset shows cumulative release of DEX from DEX-oxSWNHs/TBPNHBP on Ti plates in PBS (blue line), RPMI medium $/ 5 \%$ FBS (green line), and $\alpha-M E M / 5 \%$ FBS (red line) at $37^{\circ} \mathrm{C}$ for 24 hours. Error bars indicate standard deviation $(n=3)$. Abbreviations: DEX, dexamethasone; PBS, phosphate-buffered saline; RPMI, Roswell Park Memorial Institute; $\alpha-M E M, \alpha$-minimal essential medium; oxSWNHs, oxidized single-walled carbon nanohorns; TBP, Ti-binding peptide; NHBP-I, SWNHbinding peptide.
Various nanomaterials, including carbon nanotubes (CNTs), multi-walled CNTs, and SWNHs, promote bone formation by interacting with osteoblasts. ${ }^{55-57}$ In the present work, we combined the TBP-NHBP peptide with oxSWNHs to obtain dispersed oxSWNHs/TBP-NHBP complexes, which were adsorbed onto the Ti surface. Incorporation of the TBP-NHBP peptide conferred the binding characteristics of minTBP-1 to oxSWNHs and mediated their uniform bidimensional adsorption onto the Ti surface. NHBP-1 binds to oxSWNHs, but has low solubility, whereas fusion

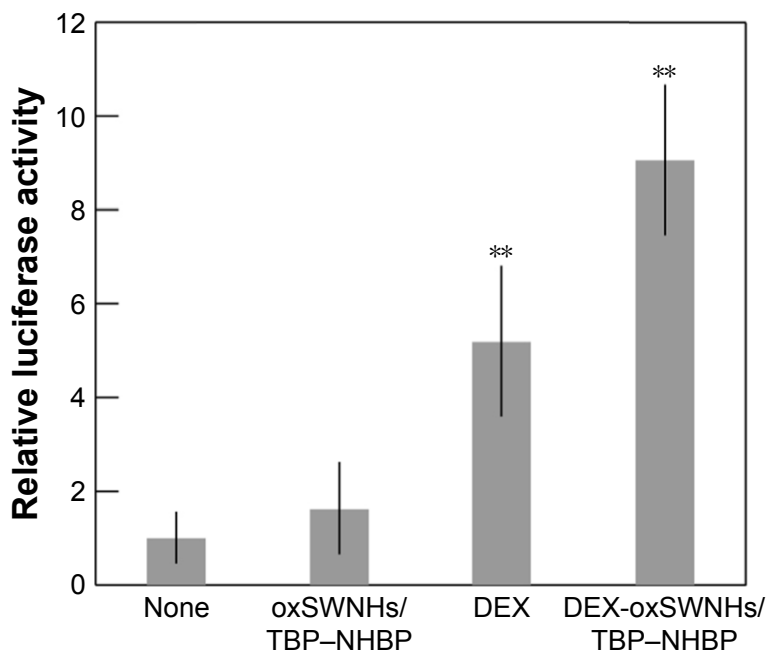

Figure 6 Effects of DEX-oxSWNHs on GR transcriptional activity. ST2 cells were transfected with pBV2-MMTV-LUC and incubated with oxSWNHs/TBP-NHBP, DEX, or DEX-oxSWNHs/TBP-NHBP on Ti plates. Error bars indicate standard deviation $(n=5)$. $* * P<0.01$.

Abbreviations: oxSWNHs, oxidized single-walled carbon nanohorns; TBP, Tibinding peptide; NHBP-I, SWNH-binding peptide; DEX, dexamethasone. 


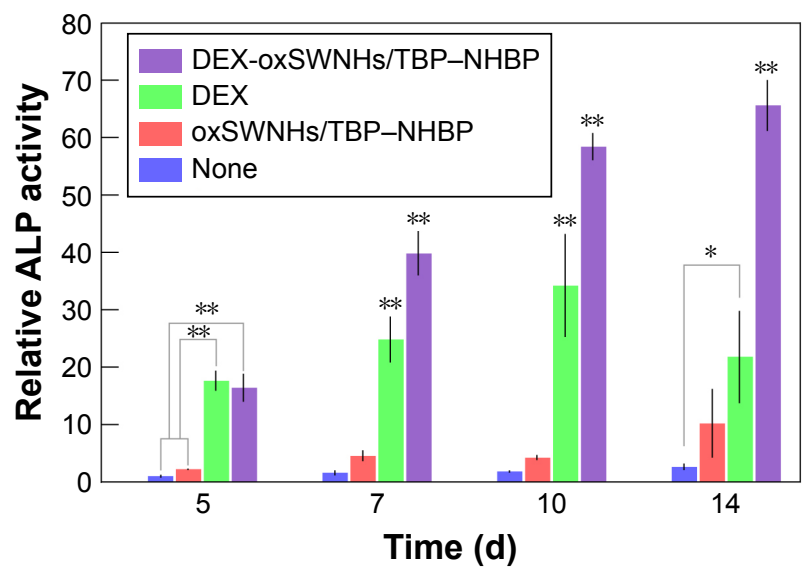

Figure 7 Effects of DEX-oxSWNHs on ALP activity. MC3TS-EI cells were cultured with oxSWNHs/TBP-NHBP, DEX, or DEX-oxSWNHs/TBP-NHBP on Ti plates for $5,7,10$, and 14 days. Error bars indicate standard deviation $(n=5)$. $* * P<0.01$; $* P<0.05$.

Abbreviations: ALP, alkaline phosphatase; DEX, dexamethasone; oxSWNHs, oxidized single-walled carbon nanohorns; TBP, Ti-binding peptide; NHBP-I, SWNHbinding peptide.

with highly soluble minTBP-1 enhances the dispersibility of oxSWNHs. This is the likely reason for the uniform adsorption of oxSWNHs onto the Ti surface without aggregation. We also showed that oxSWNHs/TBP-NHBP were adsorbed onto the Ti surface by monitoring the changes in $f$ and $D$ in a Ti plate, observing that pre-mixing TBP-NHBP and oxSWNHs was important for oxSWNHs adsorption. The decrease in $f$ and increase in $D$ were not saturated within the observation range, suggesting that surface treatment can be readily controlled. Furthermore, oxSWNHs/TBP-NHBP immobilized on $\mathrm{Ti}$ were nontoxic to cultured cells, even after 2 days of culture and in contrast with CNTs. ${ }^{58}$ SWNHs contain no metal impurities, because SWNHs are synthesized through laser ablation or arc discharge using pure graphite rods without any metal catalysts. ${ }^{59,60}$ Therefore, SWNHs have negligible toxicity both in vitro and in vivo, ${ }^{33,42,43,61}$ which facilitates fabrication of the functionalized nanoplatforms for biomedical applications with low toxicity.

To assess the potential of oxSWNHs on Ti for drug delivery, we compared the release of the anti-inflammatory glucocorticoid DEX in PBS, $\alpha$-MEM, and RPMI medium at $37^{\circ} \mathrm{C}$. DEX was released from Ti at a slower rate in $\alpha$-MEM and RPMI than in PBS; moreover, in cell-culture media, the release was sustained for up to 14 days. Variable solubility of DEX in PBS and cell-culture media could account for the differences in the release profiles. Additionally, GREs were activated by DEX and especially DEX-oxSWNHs/ TBP-NHBP, with adsorption of the latter onto Ti increasing ALP activity in MC3T3-E1 cells relative to control samples on day 14. Therefore, DEX was not delivered in a single burst at the implantation site, but was steadily released for a sustained effect.

The results of the in vitro assays indicated that DEX released from DEX-oxSWNHs on Ti plates was biologically active. To date, there have been no studies on the effectiveness of DEX delivered by oxSWNHs/TBP-NHBP on Ti for inducing osteogenesis. The majority of studies on Ti-implantsurface treatments have focused on physicochemical surface modifications to improve osseointegration and interactions between surfaces and tissues. In the Ti-surface-modification method that has been reported, a special apparatus, such as a plasma spray apparatus, was required. However, the method described in this study constitutes a simple surfacereforming method that mixes oxSWNHs, TBP-NHBP, and a drug together, followed by incubation of Ti with the mixed solution, which enables it to transport the function of the drug. Because oxSWNHs are capable of transporting various drugs, we believe that it will be possible to perform surface modifications of Ti by enabling the drug to support oxSWNHs/TBP-NHBP according to the clinical purpose. Our findings have provided a basis for the development of new biomaterials for use in dental implants and as osteoblast scaffolds for bone regeneration.

\section{Conclusion}

We have successfully developed a solid-phase drug-delivery system where oxSWNHs were immobilized on a base material using TBP-NHBP, a bispecific material-binding peptide combining functions of minTBP-1 and NHBP-1. Through the use of TBP-NHBP, oxSWNHs, which otherwise could not be attached to Ti, were successfully immobilized on the Ti surface. SEM was used to confirm the presence of oxSWNHs adsorbed onto the Ti surface, and a QCM was used to evaluate the binding process during oxSWNH adsorption. Moreover, we were able to impart the function of sustained drug release to a substrate by using oxSWNHs. The oxSWNHs/TBP-NHBP-immobilized Ti surface was nontoxic to cells and could be used to deliver DEX, an anti-inflammatory agent, over a sustained period. The released DEX was confirmed as biologically active, suggesting the potential use of oxSWNHs/TBP-NHBP as a drug-delivery system to stimulate osteogenesis and bone regeneration. Therefore, our findings suggested that surface modification is a useful approach for designing medical biomaterials with desirable properties and possible practical applications. 


\section{Acknowledgments}

We thank Dr T Imamura for kindly providing the MC3T3-E1 cells and ST2 cells, Dr K-I Sano for valuable discussion, and Ms T Minamisawa for technical assistance.

This work was supported by a JSPS KAKENHI (grant no 21791959, 16K11524).

\section{Disclosure}

The authors report no conflicts of interest in this work.

\section{References}

1. Jenny G, Jauernik J, Bierbaum S, et al. A systematic review and metaanalysis on the influence of biological implant surface coatings on periimplant bone formation. J Biomed Mater Res A. 2016;104(11): 2898-2910.

2. Kokubun K, Kashiwagi K, Yoshinari M, Inoue T, Shiba K. Motifprogrammed artificial extracellular matrix. Biomacromolecules. 2008;9(11):3098-3105.

3. Kashiwagi K, Tsuji T, Shiba K. Directional BMP-2 for functionalization of titanium surfaces. Biomaterials. 2009;30(6):1166-1175.

4. Yuasa K, Kokubu E, Kokubun K, et al. An artificial fusion protein between bone morphogenetic protein 2 and titanium-binding peptide is functional in vivo. J Biomed Mater Res A. 2014;102(4): 1180-1186.

5. Schuler M, Trentin D, Textor M, Tosatti SGP. Biomedical interfaces: titanium surface technology for implants and cell carriers. Nanomedicine. 2006;1(4):449-463.

6. Brunette DM, Tengvall P, Textor M, Thomsen P. Titanium in Medicine: Material Science, Surface Science, Engineering, Biological Responses and Medical Applications. Berlin: Springer Verlag; 2001.

7. Amigó V, Salvador MD, Romero F, Solves C, Moreno JF. Microstructural evolution of Ti-6Al-4V during the sintering of microspheres of Ti for orthopedic implants. J Mater Process Technol. 2003;141(1):117-122.

8. Kim HM, Miyaji F, Kokubo T, Nakamura T. Effect of heat treatment on apatite-forming ability of Ti metal induced by alkali treatment. J Mater Sci Mater Med. 1997;8(6):341-347.

9. Popa C, Simon V, Vida-Simiti I, Batin G, Candea V, Simon S. Titaniumhydroxyapatite porous structures for endosseous applications J Mater Sci Mater Med. 2005;16(12):1165-1171.

10. Rautray TR, Narayanan R, Kwon T-Y, Kim K-H. Surface modification of titanium and titanium alloys by ion implantation. J Biomed Mater Res B Appl Biomater. 2010;93(2):581-591.

11. Nayab SN, Jones FH, Olsen I. Effects of calcium ion-implantation of titanium on bone cell function in vitro. J Biomed Mater Res A. 2007; 83(2):296-302.

12. Shen $\mathrm{X}, \mathrm{Hu} \mathrm{Y}, \mathrm{Xu} \mathrm{G}$, et al. Regulation of the biological functions of osteoblasts and bone formation by $\mathrm{Zn}$-incorporated coating on microrough titanium. ACS Appl Mater Interfaces. 2014;6(18):16426-16440.

13. Qiao Y, Zhang W, Tian P, et al. Stimulation of bone growth following zinc incorporation into biomaterials. Biomaterials. 2014;35(25): 6882-6897.

14. Liang C, Wang H, Yang J, et al. Femtosecond laser-induced micropattern and $\mathrm{Ca} / \mathrm{P}$ deposition on Ti implant surface and its acceleration on early osseointegration. ACS Appl Mater Interfaces. 2013;5(16): 8179-8186.

15. Kazemzadeh-Narbat M, Kindrachuk J, Duan K, Jenssen H, Hancock REW, Wang R. Antimicrobial peptides on calcium phosphatecoated titanium for the prevention of implant-associated infections Biomaterials. 2010;31(36):9519-9526.

16. Radin S, Ducheyne P. Controlled release of vancomycin from thin sol-gel films on titanium alloy fracture plate material. Biomaterials. 2007;28(9):1721-1729.
17. Aninwene GE 2nd, Yao C, Webster TJ. Enhanced osteoblast adhesion to drug-coated anodized nanotubular titanium surfaces. Int J Nanomedicine. 2008;3(2):257-264.

18. Antoci V Jr, King SB, Jose B, et al. Vancomycin covalently bonded to titanium alloy prevents bacterial colonization. J Orthop Res. 2007; 25(7):858-866.

19. Mani G, Johnson DM, Marton D, et al. Drug delivery from gold and titanium surfaces using self-assembled monolayers. Biomaterials. 2008;29(34):4561-4573.

20. Zavgorodniy AV, Borrero-López O, Hoffman M, LeGeros RZ, Rohanizadeh R. Characterization of the chemically deposited hydroxyapatite coating on a titanium substrate. J Mater Sci Mater Med. 2011; 22(1):1-9.

21. Choi J, Konno T, Takai M, Ishihara K. Controlled drug release from multilayered phospholipid polymer hydrogel on titanium alloy surface. Biomaterials. 2009;30(28):5201-5208.

22. Pantarotto D, Partidos CD, Graff R, et al. Synthesis, structural characterization, and immunological properties of carbon nanotubes functionalized with peptides. J Am Chem Soc. 2003;125(20):6160-6164.

23. Mattson MP, Haddon RC, Rao AM. Molecular functionalization of carbon nanotubes and use as substrates for neuronal growth. $J \mathrm{Mol}$ Neurosci. 2000;14(3):175-182.

24. Chen RJ, Bangsaruntip S, Drouvalakis KA, et al. Noncovalent functionalization of carbon nanotubes for highly specific electronic biosensors. Proc Natl Acad Sci U S A. 2003;100(9):4984-4989.

25. Pantarotto D, Partidos CD, Hoebeke J, et al. Immunization with peptidefunctionalized carbon nanotubes enhances virus-specific neutralizing antibody responses. Chem Biol. 2003;10(10):961-966.

26. Miyako E, Deguchi T, Nakajima Y, et al. Photothermic regulation of gene expression triggered by laser-induced carbon nanohorns. Proc Natl Acad Sci U S A. 2012;109(19):7523-7528.

27. Miyako E, Kono K, Yuba E, Hosokawa C, Nagai H, Hagihara Y. Carbon nanotube-liposome supramolecular nanotrains for intelligent moleculartransport systems. Nat Commun. 2012;3:1226.

28. Murakami T, Ajima K, Miyawaki J, Yudasaka M, Iijima S, Shiba K. Drug-loaded carbon nanohorns: adsorption and release of dexamethasone in vitro. Mol Pharm. 2004;1(6):399-405.

29. Hirata E, Miyako E, Hanagata N, et al. Carbon nanohorns allow acceleration of osteoblast differentiation via macrophage activation. Nanoscale. 2016;8(30):14514-14522.

30. Kasai T, Matsumura S, Iizuka T, et al. Carbon nanohorns accelerate bone regeneration in rat calvarial bone defect. Nanotechnology. 2011; 22(6):065102.

31. Depan D, Misra RDK. The interplay between nanostructured carbongrafted chitosan scaffolds and protein adsorption on the cellular response of osteoblasts: structure-function property relationship. Acta Biomater. 2013;9(4):6084-6094.

32. Murakami T, Sawada H, Tamura G, Yudasaka M, Iijima S, Tsuchida K. Water-dispersed single-wall carbon nanohorns as drug carriers for local cancer chemotherapy. Nanomedicine (Lond). 2008;3(4): $453-463$.

33. Miyawaki J, Yudasaka M, Azami T, Kubo Y, Iijima S. Toxicity of single-walled carbon nanohorns. ACS Nano. 2008;2(2):213-226.

34. Iijima S, Yudasaka M, Yamada R, et al. Nano-aggregates of singlewalled graphitic carbon nano-horns. Chem Phys Lett. 1999;309(3-4): $165-170$.

35. Murata K, Kaneko K, Kanoh H, et al. Adsorption mechanism of supercritical hydrogen in internal and interstitial nanospaces of single-wall carbon nanohorn assembly. J Phys Chem B. 2002;106(43): 11132-11138.

36. Murata K, Kaneko K, Steele WA, et al. Molecular potential structures of heat-treated single-wall carbon nanohorn assemblies. JPhys Chem B. 2001;105(42):10210-10216.

37. Murata K, Hirahara K, Yudasaka M, Iijima S, Kasuya D, Kaneko K. Nanowindow-induced molecular sieving effect in a single-wall carbon nanohorn. J Phys Chem B. 2002;106(49):12668-12669. 
38. Yuge R, Ichihashi T, Miyawaki J, Yoshitake T, Iijima S, Yudasaka M. Hidden caves in an aggregate of single-wall carbon nanohorns found by using Gd2O3 probes. J Phys Chem C. 2009;113(7):2741-2744.

39. Murakami T, Fan J, Yudasaka M, Iijima S, Shiba K. Solubilization of single-wall carbon nanohorns using a PEG-doxorubicin conjugate. Mol Pharm. 2006;3(4):407-414.

40. Ajima K, Yudasaka M, Murakami T, Maigné A, Shiba K, Iijima S. Carbon nanohorns as anticancer drug carriers. Mol Pharm. 2005; 2(6):475-480.

41. Matsumura S, Ajima K, Yudasaka M, Iijima S, Shiba K. Dispersion of cisplatin-loaded carbon nanohorns with a conjugate comprised of an artificial peptide aptamer and polyethylene glycol. Mol Pharm. 2007; 4(5):723-729.

42. Tahara Y, Miyawaki J, Zhang M, et al. Histological assessments for toxicity and functionalization-dependent biodistribution of carbon nanohorns. Nanotechnology. 2011;22(26):265106.

43. Zhang M, Yamaguchi T, Iijima S, Yudasaka M. Size-dependent biodistribution of carbon nanohorns in vivo. Nanomed Nanotechnol Biol Med. 2013;9(5):657-664.

44. Sano K-I, Shiba K. A hexapeptide motif that electrostatically binds to the surface of titanium. J Am Chem Soc. 2003;125(47):14234-14235.

45. Kase D, Kulp JL, Yudasaka M, Evans JS, Iijima S, Shiba K. Affinity selection of peptide phage libraries against single-wall carbon nanohorns identifies a peptide aptamer with conformational variability. Langmuir. 2004;20(20):8939-8941.

46. Hayashi T, Sano K-I, Shiba K, Iwahori K, Yamashita I, Hara M. Critical amino acid residues for the specific binding of the Ti-recognizing recombinant ferritin with oxide surfaces of titanium and silicon. Langmuir. 2009;25(18):10901-10906

47. Sano K-I, Ajima K, Iwahori K, et al. Endowing a ferritin-like cage protein with high affinity and selectivity for certain inorganic materials. Small. 2005;1(8-9):826-832.

48. Sano K-I, Sasaki H, Shiba K. Utilization of the pleiotropy of a peptidic aptamer to fabricate heterogeneous nanodot-containing multilayer nanostructures. J Am Chem Soc. 2006;128(5):1717-1722.

49. Fan J, Yudasaka M, Miyawaki J, Ajima K, Murata K, Iijima S. Control of hole opening in single-wall carbon nanotubes and singlewall carbon nanohorns using oxygen. $J$ Phys Chem B. 2006;110(4): $1587-1591$
50. Abe I, Umehara K, Morita R, Nemoto K, Degawa M, Noguchi H. Green tea polyphenols as potent enhancers of glucocorticoid-induced mouse mammary tumor virus gene expression. Biochem Biophys Res Commun. 2001;281(1):122-125.

51. Yamamoto KR. Steroid receptor regulated transcription of specific genes and gene networks. Аnnu Rev Genet. 1985;19:209-252.

52. Diefenderfer DL, Osyczka AM, Garino JP, Leboy PS. Regulation of BMP-induced transcription in cultured human bone marrow stromal cells. J Bone Joint Surg Am. 2003;85-A (Suppl 3):S19-S28.

53. Luppen CA, Smith E, Spevak L, Boskey AL, Frenkel B. Bone morphogenetic protein-2 restores mineralization in glucocorticoid-inhibited MC3T3-E1 osteoblast cultures. J Bone Miner Res. 2003;18(7): $1186-1197$.

54. Luppen CA, Leclerc N, Noh T, et al. Brief bone morphogenetic protein 2 treatment of glucocorticoid-inhibited MC3T3-E1 osteoblasts rescues commitment-associated cell cycle and mineralization without alteration of Runx2. J Biol Chem. 2003;278(45):44995-45003.

55. Shimizu M, Kobayashi Y, Mizoguchi T, et al. Carbon nanotubes induce bone calcification by bidirectional interaction with osteoblasts. Adv Mater. 2012;24(16):2176-2185.

56. Saito N, Usui Y, Aoki K, et al. Carbon nanotubes for biomaterials in contact with bone. Curr Med Chem. 2008;15(5):523-527.

57. Misra RD, Chaudhari PM. Osteoblasts response to nylon 6,6 blended with single-walled carbon nanohorn. J Biomed Mater Res A. 2013; 101(4):1059-1068.

58. Shvedova AA, Castranova V, Kisin ER, et al. Exposure to carbon nanotube material: assessment of nanotube cytotoxicity using human keratinocyte cells. $J$ Toxicol Environ Health A. 2003;66(20): 1909-1926.

59. Kasuya D, Yudasaka M, Takahashi K, Kokai F, Iijima S. Selective production of single-wall carbon nanohorn aggregates and their formation mechanism. J Phys Chem B. 2002;106(19):4947-4951.

60. Li N, Wang Z, Zhao K, Shi Z, Gu Z, Xu S. Synthesis of single-wall carbon nanohorns by arc-discharge in air and their formation mechanism. Carbon. 2010;48(5):1580-1585.

61. Jiang B-P, Hu L-F, Shen X-C, et al. One-step preparation of a watersoluble carbon nanohorn/phthalocyanine hybrid for dual-modality photothermal and photodynamic therapy. ACS Appl Mater Interfaces. 2014;6(20):18008-18017.
International Journal of Nanomedicine

\section{Publish your work in this journal}

The International Journal of Nanomedicine is an international, peerreviewed journal focusing on the application of nanotechnology in diagnostics, therapeutics, and drug delivery systems throughout the biomedical field. This journal is indexed on PubMed Central, MedLine, CAS, SciSearch ${ }^{\circledR}$, Current Contents ${ }^{\circledR} /$ Clinical Medicine,

\section{Dovepress}

Journal Citation Reports/Science Edition, EMBase, Scopus and the Elsevier Bibliographic databases. The manuscript management system is completely online and includes a very quick and fair peer-review system, which is all easy to use. Visit http://www.dovepress.com/ testimonials.php to read real quotes from published authors. 